\title{
REGULATORY AND LEGAL COMPONENT IN ENSURING STATE'S FINANCIAL SECURITY
}

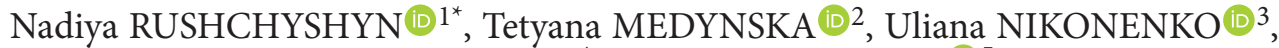 \\ Zoryana $\mathrm{KOSTAK}^{4}$, Roksolana IVANOVA (1D) 5 \\ 1,2 Lviv University of Trade and Economics, Lviv, Ukraine \\ ${ }^{3}$ Ukrainian Academy of Printing, Lviv, Ukraine \\ ${ }^{4}$ Department of Finance, Accounting and Reporting of Medenychi United Territorial Community, Lviv, Ukraine \\ ${ }^{5}$ Khmelnytskyi University of Management and Law, Khmelnytskyi, Ukraine
}

Received 20 September 2020; accepted 27 May 2021

\begin{abstract}
The purpose of the study is to scientifically substantiate the place and role of regulatory support in the formation of financial security, study the factors influencing its level and identify ways to improve it. The study used general scientific and special research methods: analysis, synthesis, methods of systematization and logical generalization - when disclosing the theoretical provisions of the financial security of the state and its legal regulation; correlation and regression analysis, econometric methods and models - to determine and study the influence of factor values on the volume of gold and foreign exchange reserves of the NBU; method of expert assessments - when developing recommendations for the formation of an effective mechanism for ensuring the financial security of the state, a graphical method. The nature and significance of the legal support of financial security are outlined, which is aimed at creating an improved legal framework and a system for controlling financial business entities in order to detect and prevent financial crimes. The factors influencing the level of the state's financial security are considered. Their influence on the volume of gold and exchange reserves of the National Bank of Ukraine which in the conditions of financial stability should be balanced is determined. The mechanism to ensure the state's financial security is offered, which further contributes to the implementation of a set of legal, structural and organizational, procedural, personnel, technological and resource tasks. In the future special attention should be paid to improving the current legal framework that would protect the interests of entities of a financial system from illegal encroachment and help recover offense damages. All these measures will contribute to raising the level of the state's financial security and will help integrate the state into the international financial and economic space.
\end{abstract}

Keywords: state's financial security, normative and legal regulation, short-term debt of Ukraine, gold and exchange reserves of the National Bank, financial stability.

JEL Classification: P48, E58, G01.

\section{Introduction}

In modern conditions of development of the Ukrainian economy, ensuring financial security is possible on the basis of systematic management of the financial and economic resources of the state, regions, enterprises. The effective functioning of enterprises is due to the stability of their financial and economic system, the ability to resist external threats in the field of finance of the real sector of the economy, therefore, it is relevant to form a mechanism for managing financial security.

The important task of the state in the context of its financial security is to create the economic and legal environment and institutional infrastructure to stimulate innovation and investment processes in the country, and at the same time to minimize the risks of all sub-systems of financial security and on that basis to contribute to the development of the state's economy in new legal conditions. Given the above mentioned, the issue of scientific and practical significance of the legal support of financial security is getting extremely important, which facilitates the creation of appropriate conditions of the financial system and prevents the abuse of funds misuse. In modern conditions of Ukraine's integration into the international community, the impact of financial offenses on the financial security of the state is significantly updated. At the same time, financial security is provided

*Corresponding author. E-mail: tulenkov_my@ukr.net

Copyright $\odot 2021$ The Author(s). Published by Vilnius Gediminas Technical University

This is an Open Access article distributed under the terms of the Creative Commons Attribution License (http://creativecommons.org/licenses/by/4.0/), which permits unrestricted use, distribution, and reproduction in any medium, provided the original author and source are credited. 
mainly by legal means - financial and legal responsibility, which prevents the abuse of financial resources. By "creating appropriate conditions" I mean the formation of a reliable, effective system of financial and legal responsibility, which prevented the misuse of funds (abuse of financial resources). After all, the legal regulation of financial security provides for the creation of favorable conditions for the functioning of the financial system of the state, in which there is practically no opportunity to direct financial flows into the spheres of their use that are not fixed by legislative normative acts.

Purpose of the research is to provide scientific substantiation of the nature and role of normative and legal support in financial security formation and the study of factors influencing its level. The study covers macro scale.

\section{Literature review}

Financial security can be explained as protection of financial interests of business entities at all levels of financial relations; provision of households, enterprises, organizations and institutions, regions, industries, sectors of the state's economy with financial resources, the ability of the financial system to ensure the effective functioning of the economic system and sustainable economic growth.

During the period of globalization changes, all subjects of the macroenvironment introduce measures to minimize the risks posed by various types of threats. Identification, prevention and elimination of such threats is an important task of the state financial security system. The level of financial security of the state depends on the level of functioning and development of the economic system, including all of its components in interconnection and interdependence. Neglect of safety can lead to negative socio-economic consequences, a depressive state of the economy, an increase in the level of import dependence, bankruptcy of market entities, and the like.

The analysis of the works of national scientists gives an opportunity to emphasize the scientific importance of the works of Baranovskyi (2004), Yermoshenko (2001), who pretty thoroughly analyse and highlight the general theoretical perspective of financial security. The interrelations of security problems, the development of the financial system of the national economy and the forecasting of its main indicators were researched by the domestic scientists like Shevchenko (2009) and by the foreign authors such as Altman (1968), Beaver (1966). Quite significant research of the financial and legal responsibility in the budget process was carried out by Hetmanets (2015). The role of financial and legal responsibility in the mechanism of legal support of Ukraine's financial security was investigated by Dmytrenko (2011), Radzivil (2011). Significant contributions to the study of the problems of guaranteeing the country's financial security under the conditions of globalization have been made by the foreign scholars such as Marsh (2012), Greenidge (2012), Knedlik and von Schweinitz (2011), Reinhart (2011), Marer (2010). In particular, the study of the factors influencing the country's external debt was carried out by Lukkezen and RojasRomagosa (2013), Gebauer et al. (2017), Zaharii (2020), Kulitsky (2020), Baldacci (2010) and Bazilyuk (2020). Both foreign and domestic scholars like Stiglitz (2010), LopezClaros (2014), Benigno (2013), Krugman (2008), Roubini (2015), Vasyltsiv (2011), Helberg (2011) researched the issue of the formation of an anti-crisis policy in order to provide financial security at the macro and micro levels.

Franchuk et al. (2020) explored the main ways to counter financial security threats, but we focused more on the legal aspect and the state level.

Sylkin et al. (2019) demonstrated how crisis management can significantly contribute to ensuring financial security, when our study is more about identifying the main factors influencing its level and demonstrating the level of importance of the legal aspect on financial security.

A large number of scientific papers have been devoted to the study of the problems of ensuring the financial security of the enterprise, among which the following should be highlighted: Malik (2015) (conducted a thorough study of the evolution of scientific views on the nature of the financial security of the enterprise); Kartuzov (2012) (formed the main categories that must be attributed to the conceptual apparatus of the financial security of the enterprise); Arefieva and Kuzenko (2009) (considered financial security from the perspective of six approaches).

However, due to the constant changes in the legislative field and the strengthening of the negative influence of factors of the external and internal environment on the state's financial security, there is a need to find new tools to influence its level improvement.

The research is based on economic theory. The article is written on the basis of research by both domestic and foreign scientists, which highlighted the issues of financial security of the state and its regulatory support.

The team of authors has identified a mechanism for ensuring the financial security of the state with the definition of the place and features of the relationships between the components of this mechanism. The novelty of this article includes proposals for the creation of a coordination center that would be able to objectively and comprehensively monitor the changes occurring in the economy of the state and its financial sphere in order to identify and predict internal and external threats. It is also proposed to create institutional and legal structures that should deal with this issue in the legislative and managerial field by making changes to existing regulatory legal acts and creating new ones.

We strive to scientifically clarify the place and role of regulatory support in the formation of financial security. To develop ways to study the factors of influence on its level and determine the ways of improvement.

\section{Research model and results}

The state's financial security occupies a significant place in the system of economic security, as it covers the most 


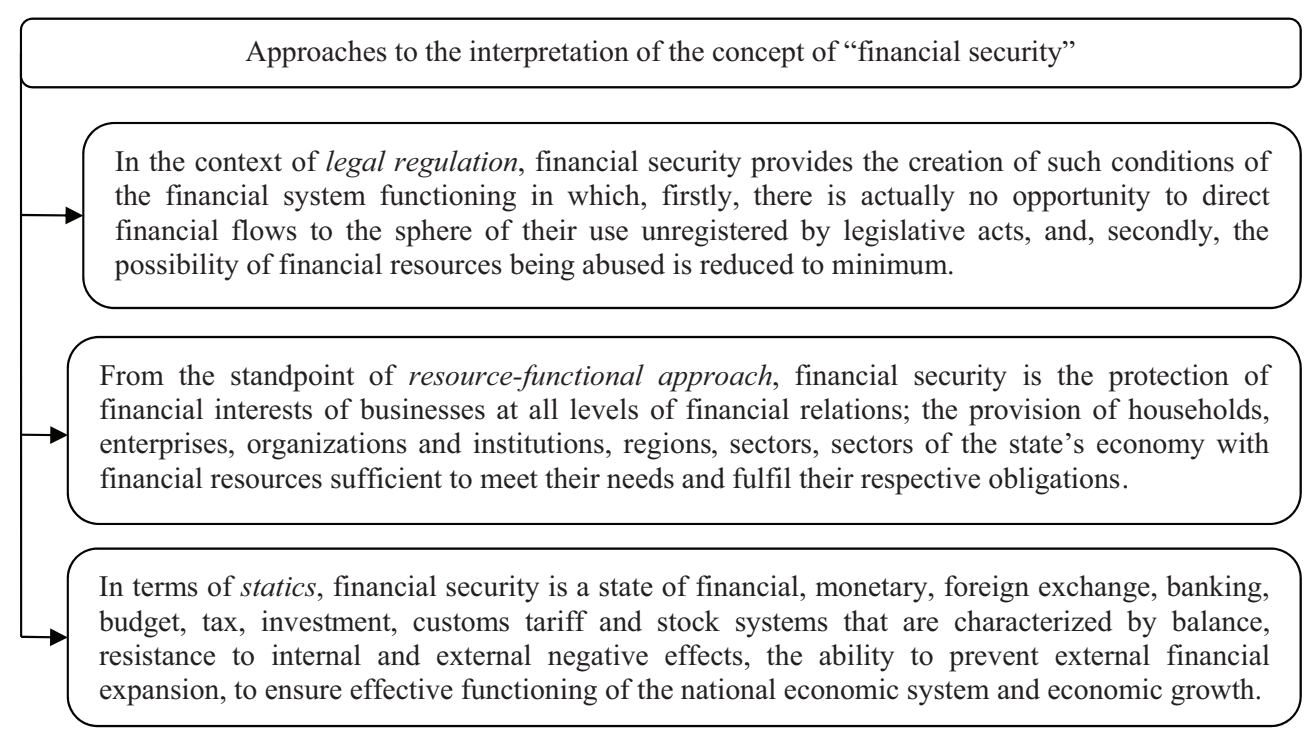

Figure 1. Approaches to the interpretation of the concept of "financial security" (source: developed by authors)

important financial issues of the national economy's entities. The formation and implementation of the mechanism to ensure the state's financial security require the consideration of this concept nature, outlining factors affecting its status, determining the relationships between the components of the structure.

Today there is no single interpretation of the concept of "financial security". National scholars (Baranovskyi, 2004; Yermoshenko, 2001; Shevchenko, 2009) consider financial security according to the different approaches presented in Figure 1.

Considering the state's financial security in the context of its legal regulation, it should be agreed with Dmytrenko (2011), who believes that, "...the essence of the legal support of financial security is the arrangement of public relations in this area, their legal consolidation and protection exercised by the state through the system of legal means". The author believes that the mechanism of legal support of financial security as a system of legal means, first of all, is aimed at creating a perfect legal framework and a financial system control in order to ensure the stability of the financial system, prevention, detection and termination of financial violations.

This makes it possible to assert that legal support in the form of different kinds of regulatory legislation will help create favourable conditions protected from the financial criminal environment to develop the state's financial sector.

From the standpoint of the resourceful approach, financial security is the protection of financial interests at all levels of financial relations; a certain degree of independence, stability and stableness of the country's financial system under the conditions of influence of external and internal destabilizing factors that pose a threat to financial security; ability of the financial system to ensure effective functioning of the national economy and sustainable economic growth.

The creation of an effective financial security system provides for a clear definition of sources of potential threats in one or another sphere, as well as available and necessary resources for their neutralization. The active factors that may pose threats to the state's financial security include: the negative dynamics of the balance of trade and balance of payment and a significant dependence of Ukraine on export and import activities; the impact of global financial crises on Ukraine's financial system of.

The definition of the volumes of gold and exchange reserves adequate to the development of the state's economy, which should be balanced with the system of financial and macroeconomic indicators at each stage of the country's development, has a sufficiently significant impact of the above-mentioned threats on the functioning of the state's financial sector and its financial security. The sufficient volume of gold and exchange reserves will ensure the state's financial stability and will help to avoid default, timely paying debts. It is possible to evaluating such a balance using econometric methods and models.

The interaction of a resulting index $(y)$ with factor variables $\left(x_{1}, x_{2}, \ldots, x_{n}\right.$. $)$ is traditionally described by linear multivariable regression:

$$
y=a_{0}+\sum_{i=m}^{n} a_{i} x_{i}
$$

The volume of the National Bank of Ukraine gold and exchange reserves was selected as the resulting index $(y)$, \$ million, and $\mathrm{X} 1-\mathrm{X} 8$ - as factor values.

It is known that a sufficient volume of international reserves is one of the factors that ensures an adequate level of financial security of the country, contributes to the stable development of the economy, increases confidence in the national currency, thereby forming a margin of financial strength in conditions of uncertainty or the impact of certain threats. In our study, it is the short-term debt of Ukraine and the balance of foreign trade operations that have the opposite effect on the resulting indicator. Therefore, a lot of efforts need to be made to ensure that, 


\begin{tabular}{|c|c|c|c|c|c|c|c|c|c|}
\hline ڤे & $\begin{array}{l}\text { ठั } \\
\text { ద్ర }\end{array}$ & $\begin{array}{l}8 \\
\text { ¿े } \\
\text { b }\end{array}$ & 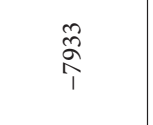 & $\vec{\Xi}$ & 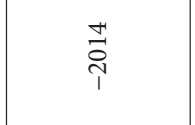 &  & $\begin{array}{l}\underset{H}{H} \\
\vec{b}\end{array}$ & 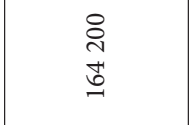 & $\begin{array}{l}\hat{\Omega} \\
\stackrel{2}{\exists}\end{array}$ \\
\hline$\stackrel{\infty}{\vec{n}}$ & 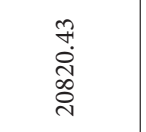 & 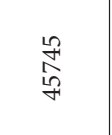 & 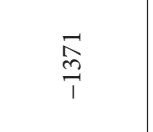 & $\stackrel{\substack{n \\
\sim}}{a}$ & 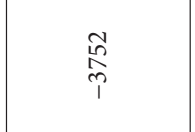 & $\stackrel{\infty}{\stackrel{\infty}{\sim}}$ & 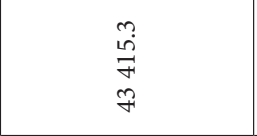 & $\begin{array}{l}\widetilde{1} \\
\infty \\
0 \\
-\end{array}$ & $\begin{array}{l}\stackrel{\hat{Q}}{2} \\
\stackrel{\Xi}{\exists}\end{array}$ \\
\hline $\overrightarrow{\text { iे }}$ & $\begin{array}{l}\not \\
\infty \\
\infty \\
\infty \\
\stackrel{\infty}{-1}\end{array}$ & $\begin{array}{l}\stackrel{\hat{N}}{6} \\
\stackrel{+}{*}\end{array}$ & $\underset{i}{\stackrel{3}{i}}$ & $\begin{array}{l}\mathbb{H} \\
\text { S }\end{array}$ & $\underset{m}{m}$ & $\begin{array}{l}0 \\
\stackrel{0}{0} \\
\text { ¿ें }\end{array}$ & 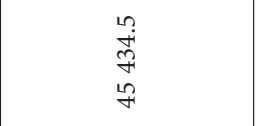 & 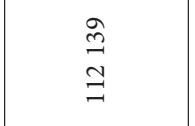 & 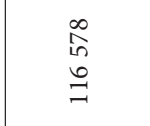 \\
\hline 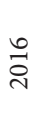 & 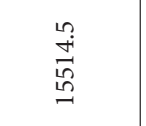 & $\begin{array}{l}\overrightarrow{\widetilde{N}} \\
\underset{f}{f}\end{array}$ & $\underset{i}{\stackrel{m}{T}}$ & $\begin{array}{l}n \\
0 \\
0 \\
0 \\
0 \\
m\end{array}$ & ल) & 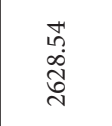 & $\begin{array}{l}\stackrel{9}{2} \\
\stackrel{+}{2} \\
\vec{F}\end{array}$ & $\begin{array}{l}\vec{\Xi} \\
\stackrel{2}{\Omega}\end{array}$ & $\begin{array}{l}\hat{B} \\
\stackrel{m}{\exists}\end{array}$ \\
\hline$\stackrel{n}{\tilde{N}}$ & $\begin{array}{l}\text { बे } \\
\text { సे }\end{array}$ & $\underset{\substack{n \\
i n}}{i n}$ & ָ̊ & 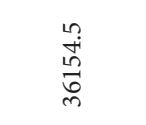 & 岂 & $\begin{array}{l}\overrightarrow{\tilde{J}} \\
\underset{\mathrm{J}}{\mathrm{N}}\end{array}$ & 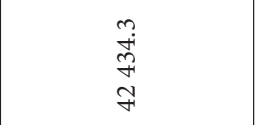 & $\begin{array}{l}\stackrel{2}{0} \\
\overrightarrow{0} \\
2\end{array}$ & 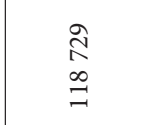 \\
\hline$\underset{\stackrel{\sim}{\sim}}{\stackrel{H}{*}}$ & 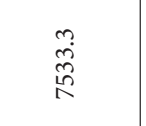 & 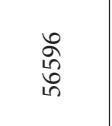 & 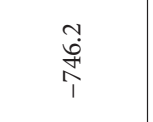 & 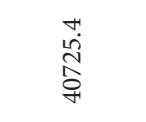 & $\overrightarrow{\widetilde{S}}$ & 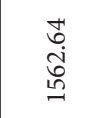 & $\begin{array}{l}\stackrel{n}{+} \\
\stackrel{+}{+} \\
\stackrel{\sigma}{0}\end{array}$ & $\begin{array}{l}\stackrel{n}{\infty} \\
\infty \\
\vec{m}\end{array}$ & 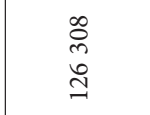 \\
\hline$\stackrel{m}{\stackrel{\sim}{~}}$ & 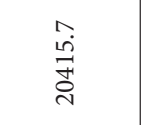 & $\begin{array}{l}\text { ते } \\
\text { ถે }\end{array}$ & 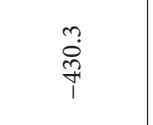 & $\underset{\substack{\stackrel{0}{0} \\
\hat{n}}}{ }$ & 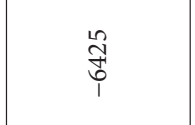 & $\begin{array}{l}\underset{+}{+} \\
\underset{\infty}{+}\end{array}$ & $\begin{array}{l}\stackrel{\sim}{\vec{m}} \\
\stackrel{m}{\Xi}\end{array}$ & $\begin{array}{l}\stackrel{0}{m} \\
\tilde{\infty} \\
\stackrel{\infty}{=}\end{array}$ & 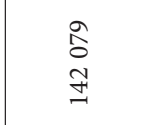 \\
\hline 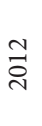 & 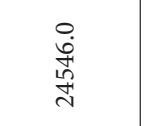 & $\begin{array}{l}n \\
0 \\
0 \\
0 \\
0\end{array}$ & $\overrightarrow{\stackrel{P}{P}}$ & $\begin{array}{l}m \\
\stackrel{2}{0} \\
\stackrel{1}{n}\end{array}$ & ڤે̀ & $\begin{array}{l}\stackrel{L}{0} \\
\stackrel{0}{\circ} \\
\stackrel{\infty}{2}\end{array}$ & $\begin{array}{l}+1 \\
0 \\
m \\
2\end{array}$ & $\begin{array}{l}\vec{\infty} \\
\stackrel{0}{\Lambda} \\
\stackrel{0}{=}\end{array}$ & 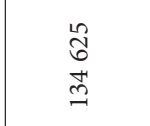 \\
\hline$\overline{\vec{\sim}}$ & $\begin{array}{l}0 \\
\dot{0} \\
\stackrel{0}{m} \\
\end{array}$ & $\begin{array}{l}\infty \\
0 \\
0 \\
0\end{array}$ & $\stackrel{\leftrightarrow}{\stackrel{\oplus}{0}}$ & $\begin{array}{l}\stackrel{0}{\dot{d}} \\
\stackrel{a}{a} \\
\stackrel{\alpha}{\sigma}\end{array}$ & $\begin{array}{l}\frac{\infty}{+} \\
\hat{i} \\
1\end{array}$ & $\begin{array}{l}\vec{a} \\
\vec{\infty}\end{array}$ & 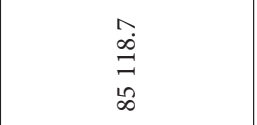 & $\begin{array}{l}\stackrel{8}{0} \\
\stackrel{0}{0}\end{array}$ & 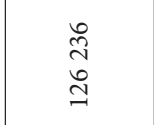 \\
\hline$\stackrel{\text { ते }}{\text {. }}$ & 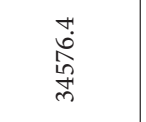 & 命 & $\overrightarrow{\hat{m}}$ & $\begin{array}{l}\stackrel{0}{0} \\
0 \\
0 \\
\stackrel{4}{+}\end{array}$ & $\stackrel{\infty}{\stackrel{一}{\Lambda}}$ & 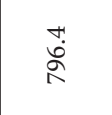 & 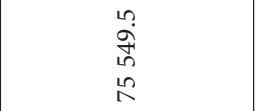 & $\begin{array}{l}\stackrel{\sigma}{7} \\
\overrightarrow{0} \\
\stackrel{0}{2}\end{array}$ & $\begin{array}{l}\stackrel{P}{N} \\
\triangleq \\
=\end{array}$ \\
\hline ڤ્ণે & 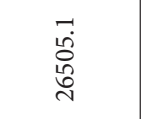 & $\stackrel{m}{\sharp}$ & $\begin{array}{l}\infty \\
\stackrel{\infty}{0} \\
i\end{array}$ & $\begin{array}{l}\vec{H} \\
\stackrel{0}{0} \\
\stackrel{0}{0} \\
m\end{array}$ & $\stackrel{\text { oे }}{\rightarrow}$ & $\begin{array}{l}\infty \\
\text { న̊ } \\
\end{array}$ & $\begin{array}{l}n \\
\hat{0} \\
\tilde{m} \\
\vec{b}\end{array}$ & $\begin{array}{l}\stackrel{\infty}{N} \\
\triangleq \\
=\end{array}$ & $\begin{array}{l}\stackrel{0}{\infty} \\
\stackrel{m}{\varrho}\end{array}$ \\
\hline 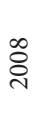 & 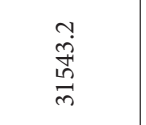 & 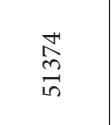 & 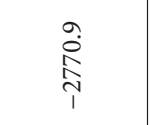 & 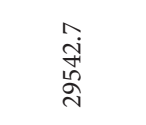 & $\begin{array}{l}\infty \\
\stackrel{\sim}{\sim} \\
\stackrel{0}{1}\end{array}$ & 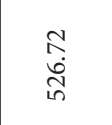 & $\begin{array}{l}\stackrel{0}{i} \\
\stackrel{1}{-} \\
\hat{6}\end{array}$ & $\begin{array}{l}\text { สิ } \\
\text { ปે }\end{array}$ & $\begin{array}{l}\hat{b} \\
\overrightarrow{0}\end{array}$ \\
\hline ڤ્ণે & 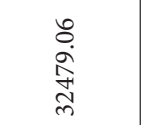 & 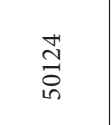 & $\begin{array}{l}\stackrel{H}{0} \\
\stackrel{1}{1}\end{array}$ & $\begin{array}{l}\stackrel{n}{?} \\
\stackrel{0}{0} \\
\stackrel{\sim}{d}\end{array}$ & 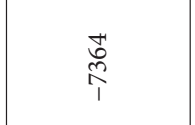 & $\begin{array}{l}\infty \\
\infty \\
\vec{D}^{+} \\
\text {in }\end{array}$ & 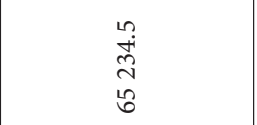 & 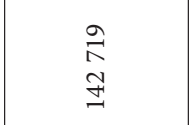 & $\begin{array}{l}\hat{\kappa} \\
\hat{\alpha} \\
\alpha\end{array}$ \\
\hline ¿্ণ & 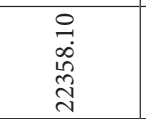 & ڤ్̊ి & 仓̊ํํ & $\begin{array}{l}\stackrel{0}{0} \\
\stackrel{0}{0}\end{array}$ & 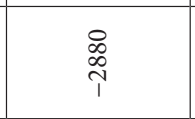 & $\begin{array}{l}8 \\
\text { in } \\
\text { in }\end{array}$ & $\begin{array}{l}\hat{y} \\
\text { ڤे } \\
\text { oे } \\
\text { in }\end{array}$ & $\begin{array}{l}\hat{n} \\
\stackrel{2}{\wedge} \\
\stackrel{0}{0}\end{array}$ & $\begin{array}{l}\text { I } \\
\text { in } \\
\stackrel{H}{n}\end{array}$ \\
\hline ્ֻণి & 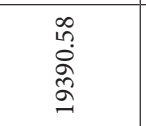 & $\begin{array}{l}\text { ஓे } \\
\text { హ̆ }\end{array}$ & $\vec{\infty}$ & $\begin{array}{l}\stackrel{0}{0} \\
\stackrel{\hat{े}}{2}\end{array}$ & 导 & $\begin{array}{l}\text { fे } \\
\text { in }\end{array}$ & $\begin{array}{l}0 \\
0 \\
0 \\
1 \\
0 \\
0 \\
n\end{array}$ & 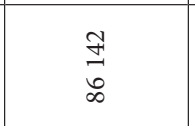 & $\begin{array}{l}\hat{\sigma} \\
\hat{m}\end{array}$ \\
\hline 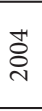 & 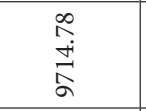 & $\begin{array}{l}\text { के } \\
\text { o. } \\
\text { \& }\end{array}$ & $\stackrel{n}{9}$ & \begin{tabular}{l}
+ \\
\multirow{2}{*}{} \\
దे
\end{tabular} & 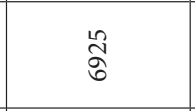 & $\frac{\tilde{\sigma}}{\tilde{n}}$ & $\begin{array}{l}0 \\
\dot{0} \\
\infty \\
i n \\
i n\end{array}$ & $\begin{array}{l}\infty \\
\infty \\
\infty \\
b \\
\\
\end{array}$ & $\begin{array}{l}\hat{f} \\
\text { bे } \\
\text { D }\end{array}$ \\
\hline 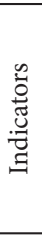 & 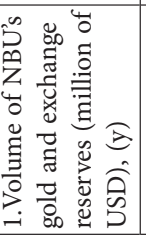 & 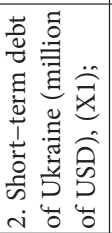 & 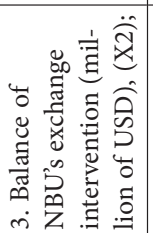 & 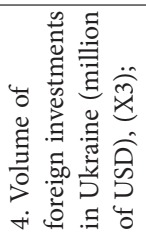 & 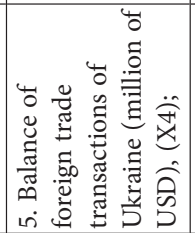 & 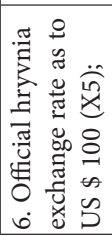 & 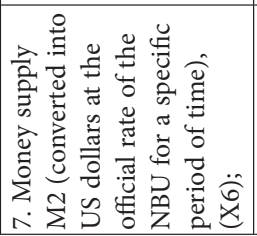 & 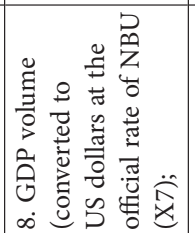 & 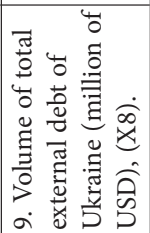 \\
\hline
\end{tabular}


in conditions of economic instability, and as a result of the negative impact of COVID-19, the volume of foreign exchange reserves does not decrease, but is sufficient to cover debts.

The summarized data for correlation and regression analysis of the influence of factor values on the volume of gold and exchange reserves of the National Bank of Ukraine are given in Table 1.

As a result of the task solution of the eight factor correlation-regression analysis, an economic and mathematical model is compiled, which has the following form:

$$
\begin{aligned}
& Y=75500.5-0.58 X 1+0.98 \mathrm{X} 2-0.05 \mathrm{X} 3- \\
& 2.03 \mathrm{X} 4-13.3 \mathrm{X} 5-0.12 \mathrm{X} 6-0.31 \mathrm{X} 7+0.38 \mathrm{X} 8 . \\
& \begin{array}{ccccc}
(3.54) & (-1.5) & (0.35) & (-0.2) \\
(-2.90) & (-2.05) & (-0.65) & (-1.49) & (1.81)
\end{array}
\end{aligned}
$$

As $F_{\text {calcul }}(7.373)>F_{\text {table }}(4.82)$, then with reliability $p=0.95$ it can be assumed that the resulting mathematical model is adequate to the experimental data (Table 2).

Table 2. Indicators characterizing the reliability of the regression model (source: developed by authors)

\begin{tabular}{|l|c|c|c|c|c|}
\hline & df & SS & MS & F & $\begin{array}{l}\text { F Signi- } \\
\text { ficance }\end{array}$ \\
\hline $\begin{array}{l}\text { Reg- } \\
\text { ression }\end{array}$ & 8 & 825700931.8 & 103212616.5 & 7.373 & 0.009 \\
\hline $\begin{array}{l}\text { Ba- } \\
\text { lance }\end{array}$ & 7 & 165203169.4 & 23600452.7 & & 0.991 \\
\hline Sum & 15 & 990904101.2 & & & \\
\hline
\end{tabular}

The value of the multiplier correlation coefficient $\mathrm{R}=$ 0.913 (Table 3) indicates a close correlation between the volume of the National Bank of Ukraine (NBU) gold and exchange reserves and a short-term debt, a foreign trade balance, the hryvnia official exchange rate, etc.
Table 3. Indicators of regression statistics

\begin{tabular}{|l|c|}
\hline \multicolumn{1}{|c|}{ Indicators of regression statistics } & Calculated values \\
\hline Multiplier regression coefficient $\mathrm{R}$ & 0.913 \\
\hline Determination coefficient $\mathrm{R}^{2}$ & 0.833 \\
\hline $\begin{array}{l}\text { Normalized coefficient of } \mathrm{R}^{2} \\
\text { determination }\end{array}$ & 0.643 \\
\hline Standard error & 4858.03 \\
\hline
\end{tabular}

The value of determination coefficient $\mathrm{R}^{2}=0.833$ shows that $83.3 \%$ of the variance or change in the average volume of NBU's gold and exchange reserves depends on changes in the balance of foreign exchange interventions, the volume of foreign investment, the hryvnia's official exchange rate and other factors combined.

The formation of reliable financial security is preceded by the creation of the necessary amount of resources to neutralize certain risks (threats). Such a resource in modern conditions is a sufficient amount of gold and foreign exchange reserves.

The estimation of the economic and mathematical model coefficients by the Student's $\mathrm{t}$-criterion shows that the following factors of the selected eight ones have a statistically probable influence:

- short-term debt of Ukraine - under the increase of this factor by 1 million USD, the volume of NBU's gold and exchange reserves will decrease by 0.58 million USD;

- balance of Ukraine's foreign trade transactions - under the increase of this factor by 1 million USD, the volume of NBU's gold and exchange reserves will decrease by 2.03 million USD;

- hryvnia's official rate - under the increase of this factor by 1 million USD, the volume of NBU's gold and exchange reserves will decrease by 13.3 million USD;

- money supply M2 - under the increase of this factor by 1 million USD, the volume of money supply will decrease by 0.12 million USD.

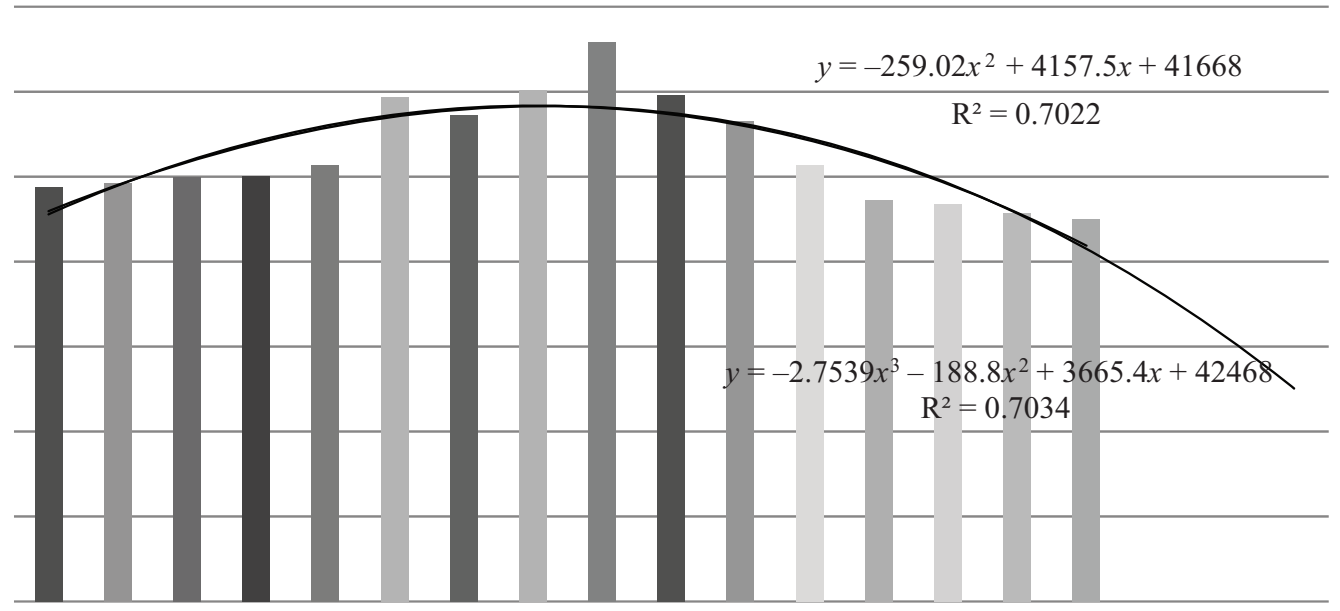

च Ukraine's short-term debt, USD million, X1

Note: $y=-259.02 x+4157.5 x+41668=51809.32$ million USD.

Figure 2. Dynamics of the Ukraine's short-term debt for 2004-2019, USD million and the line-of-trend equation by the method of the least squares (source: developed by authors) 
Having determined the significant impact of the Ukraine's short-term debt on the performance indicator the volume of the NBU's gold and exchange reserves, the authors will make this indicator forecast for three years. To do this, the authors will make up a trend model and define the trend equation using the method of the least squares (Figure 2). Determination coefficient $\left(\mathrm{R}^{2}=0.7022\right)$ has an approximate value of one. This suggests that the parabolic trend model for Ukraine's short-term debt has a statistical probability.

Based on the equation depicted in Figure 2, the forecast of Ukraine's short-term debt for 2022 is calculated: Thanks to the trend analysis it is determined that the

Table 4. Manifestations of negative risk effects (source: developed by authors)

\begin{tabular}{|c|c|}
\hline $\begin{array}{l}\text { Subjects } \\
\text { subject to } \\
\text { the negative } \\
\text { impact of } \\
\text { risks }\end{array}$ & Manifestations of the negative impact of risks \\
\hline \multirow{5}{*}{$\begin{array}{l}\text { For } \\
\text { individuals, } \\
\text { citizens }\end{array}$} & $\begin{array}{l}\text { Exemption from enterprises, organizations, } \\
\text { institutions whose activities are suspended as a } \\
\text { result of quarantine }\end{array}$ \\
\hline & $\begin{array}{l}\text { Transfer of employees to vacations at their } \\
\text { own expense, to reduced wages, etc., with a } \\
\text { corresponding loss of income }\end{array}$ \\
\hline & $\begin{array}{l}\text { Chain of layoffs of workers in related sectors } \\
\text { due to reduced demand for their products }\end{array}$ \\
\hline & Critical decline in household income \\
\hline & $\begin{array}{l}\text { Increasing prices for important goods and } \\
\text { services and / or limiting the possibilities for } \\
\text { their uninterrupted supply }\end{array}$ \\
\hline \multirow{5}{*}{$\begin{array}{l}\text { For small } \\
\text { and } \\
\text { medium } \\
\text { businesses }\end{array}$} & $\begin{array}{l}\text { Dissolution of traditional supply chains, as a } \\
\text { result - lack of materials and components }\end{array}$ \\
\hline & $\begin{array}{l}\text { A tangible decrease in demand and loss of sales } \\
\text { markets }\end{array}$ \\
\hline & $\begin{array}{l}\text { Lack of labor force, incl. due to the inability of } \\
\text { employees to travel }\end{array}$ \\
\hline & $\begin{array}{l}\text { Increase in accounts receivable and payable, } \\
\text { shortage of working capital, and, as a result, } \\
\text { deterioration of the financial condition of } \\
\text { enterprises }\end{array}$ \\
\hline & $\begin{array}{l}\text { The takeover of bankrupt companies and those } \\
\text { whose financial condition has deteriorated } \\
\text { by large monopoly enterprises, primarily } \\
\text { by vertically organized holdings (deepening } \\
\text { monopolization) }\end{array}$ \\
\hline \multirow{4}{*}{$\begin{array}{l}\text { For the } \\
\text { economic } \\
\text { security of } \\
\text { the state }\end{array}$} & $\begin{array}{l}\text { A significant increase in the volume of } \\
\text { expenditures due to the devaluation of the } \\
\text { hryvnia, an increase in the cost of servicing the } \\
\text { public debt, etc. }\end{array}$ \\
\hline & $\begin{array}{l}\text { Deterioration of the state of public finances due } \\
\text { to a decrease in state budget revenues }\end{array}$ \\
\hline & $\begin{array}{l}\text { Accumulation of accounts receivable and } \\
\text { payable of government agencies }\end{array}$ \\
\hline & $\begin{array}{l}\text { Growth in non-payments for housing } \\
\text { and communal services and difficulties in } \\
\text { the operation of utilities, deterioration of } \\
\text { infrastructure }\end{array}$ \\
\hline
\end{tabular}

regression model has a linear function and a fairly high value of the determination coefficient, and the forecast value of the Ukraine's short-term debt is calculated, which will be more on 6809.32 USD million, compared with 2019, which is a negative step for raising the level of the state's financial security in the near future.

As a result of the correlation-regression analysis of the dependence of the level of NBU's gold and exchange reserves on the selected factors, a lot of efforts are needed to reduce the Ukraine's short-term debt to ensure a stable level of financial security.

In addition, the COVID-19 pandemic will have a negative impact on the level of financial security, which is an important component of the economic security of the state. "Experts predict that Ukrainian GDP will fall more than the world one - by $4.2 \%$. At the same time, the range of estimates is very wide - from a fall of $6.6 \%$ to an increase of $1.4 \%$. But already in 2021. A gradual recovery will begin. The median forecast of analysts is $2.4 \%$ (the range is from 1.5 to $4.4 \%)$. The consensus forecast presented by experts from the Ministry of Economic Development, Trade and Agriculture, the National Bank of Ukraine, the institutes of the National Academy of Sciences of Ukraine, Gosvneshinform, the International Center for Advanced Research, Dragon Capital, ICU, VoxUkraine, provides for a reduction in consumption by $3 \%$, imports - by $12.2 \%$, exports - by $7.9 \%$, industrial production - by $6.1 \%$, agricultural - by $1.1 \%$. Experts predict inflation for 2020 at $7 \%$, and $5.9 \%$ for 2021 . The average annual exchange rate is projected at $28.85 \mathrm{UAH} / \mathrm{USD}$ in 2020 and $30.00 \mathrm{UAH} /$ USD - in 2021.”.

The total losses of the state and local budgets can reach UAH 150-200 billion. At the same time, about 600-700.000 enterprises, small entrepreneurs and educational institutions providing jobs for 3.5-4 million people have already stopped work due to quarantine. This is about $1 / 4$ of those employed in the Ukrainian economy.

We believe that due to the prolongation of quarantine measures, the negative impact of risks to the financial and economic security of individuals, legal entities and the state in general, the manifestations of which are reflected in Table 4, will increase.

It should be noted that domestic experts carried out an integral assessment of both internal and external risks that will have an impact on the development of the Ukrainian economy in 2020-2021. This integral assessment is calculated as the product of estimates of the probability of the occurrence of the phenomenon and the impact of the phenomenon on the economy, weighted by the number of respondents. The maximum integral value of both external and internal risks is 16 .

So a significant impact on the domestic economy, from internal risks, has an increase in the state budget deficit and cash gaps in the pension fund of Ukraine, other state social insurance funds (with an integral assessment of 14 points in 2020 and 13 points in 2021 from the maximum possible 16) ... In previous years, this risk was assessed by specialists as quite significant (for 2017-2019, the average 
integral estimate was 8), but it turned out to be the most likely due to the intensification of the COVID-19 pandemic. In addition to this risk, high scores (above 10) for 2020 were received by seven of the 12 first proposed internal risks, which are to some extent associated with the COVID-19 pandemic, namely: an increase in unemployment due to the return of workers, mass layoffs workers $(13 / 11)$ the spread of COVID-19, the medical system is not able to stop the pandemic in Ukraine (12/10) a significant deterioration in the quality of life of the population, especially the most vulnerable (12/8) bankruptcy of small and medium-sized businesses (12/8); acceleration of inflationary processes (11/9).

An integrated assessment of external risks (phenomena) for 2020 and 2021 gives grounds to assert their significant impact on the country's economy, especially those that are not assessed for the first time, namely: a decrease in opportunities for access to international capital and a shortage of external financing (integrated assessment for $2020-13$ for $2021-10$ (in the previous survey the integral assessment of this risk for 2020 was 8, for $2021-7$ ) narrowing of external markets for products and services due to high competition in $2020-12$ (in the previous survey 7), in 2021 - 9 (8); the world economic crisis (as a result of the destruction of established production ties, bankruptcy of a number of industrial producers in the EU / world) in $2020-11$ (8), in $2021-9$ (7). A new external risk - The second wave of the COVID-19 pandemic in the world: $11 / 7$, and the risk of refusal to cooperate with the IMF turned out to be the least significant: an integral estimate of 3 in 2020, in $2021-2$.

Based on the results of the above study, we believe that the COVID-19 pandemic in Ukraine will have a negative impact on the financial and economic development of the country's economy, in turn, may undermine its already low level of financial security. Indeed, in the second half of 2019, there was a gradual decrease in the volume of gross domestic product in the country, signs of an economic recession appeared, there was a significant drop in the activity of external investors, who, due to political instability and the continuation of hostilities in the east of the country, refused to invest their capital in the development of the economy.

To minimize the negative impact of the COVID-19 pandemic on the financial security of the state, one should systematically approach the solution of this problem, namely, skillfully combine instruments of state financial support with the organizational and economic instruments of local authorities to optimize the use of decentralized financial resources and financial resources of small and medium-sized businesses. In addition, it will be advisable to introduce legal and regulatory changes that will make it possible to protect the financial interests of business entities due to restrictions on their rights during the quarantine period.

Consequently, it can be argued that the level of debt security is low and public authorities need to take radical steps to reduce the debt dependence on international financial institutions and respond timely to offenses in the financial sector.

Financial offenses cover all the areas of the national economy, which include: offenses in the field of external and internal borrowings, which lead to the misuse of borrowed funds; the budget, tax breaches; offenses in the field of money circulation and settlements; currency violations, etc.

The analysis of financial and other kinds of legislation shows that to combat financial crime it is necessary to improve the law, including rules governing the mechanism of financial liability. It can be done through amendments to existing legislation, namely:

1. The Budget Code of Ukraine shall be supplemented with rules on: the responsibility of the Cabinet of Ministers of Ukraine, the Ministry of Finance of Ukraine, the Accounting Chamber, its officials for delays in the budget process (Articles 35, 38, 117); on the system of offenses in the field of local borrowings and liability in this area (Section V); on the liability for the illegal use of foreign currency by budgetary institutions (Chapter 18).

2. Revised Chapter 11 of the Tax Code of Ukraine called "Financial Liability for Tax Violations" shall be adopted to combine Chapters 11,12 and supplemented with Articles "Responsibility of the State Tax Service and Officials for Tax Offenses", "Exemption from Liability of the Person who Committed Tax Offenses".

3. Section X of the Law "On the National Bank of Ukraine" shall provide for a norm on the liability of the NBU, its governing bodies and officials for the use of emission funds to finance the state budget deficit. At the same time, it is necessary to elaborate drafts of individual regulations (Banking and Currency Codes, Laws of Ukraine: "On Declaring Personal Income", "On International Technical Assistance", "On the Fund for the Protection of the Rights of Individuals-Investors", "On Payment Cards", "Regulations on gold and exchange reserves of Ukraine, etc.) with the rules on the mechanism of financial and legal liability in case of committing financial offenses.

Therefore, in order to increase the level of state's financial security, it is necessary to create a mechanism for ensuring financial security, which will further help to implement a range of legal, structural, organizational, procedural, personnel, technological and resource tasks (Figure 3).

The creation of a local coordination center that would be able to objectively and thoroughly monitor the changes that arise in the state's economy and its financial sector in order to identify and predict internal and external threats should be of extreme importance.

With regard to the regulatory framework of financial security, it is recommended to create institutional and legal structures that have to deal with this issue in the legal and administrative field through amendments to existing regulations and creating new ones. 


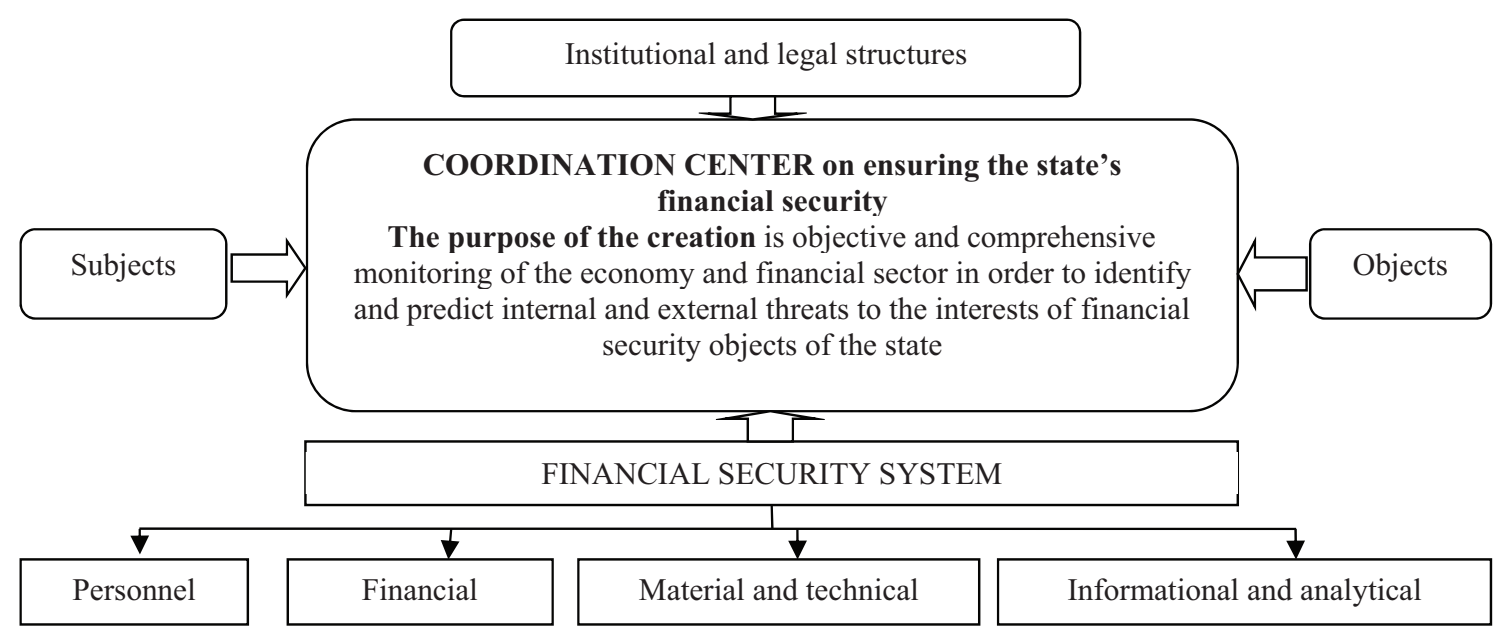

Figure 3. Mechanism of ensuring the state's financial security (developed by authors)

\section{Conclusions}

Therefore, in order to ensure an adequate level of financial security, state authorities need to make considerable efforts to form an effective mechanism for ensuring the state's financial security. It is recommended to form a coordination center to ensure financial security, which cooperating with legislative, executive and judicial branches of the government would objectively and comprehensively monitor the economy and the financial sector to identify and predict internal and external threats to the financial interests of state's financial security.

Today, the question of researching and concretizing the regulatory and legal component of ensuring financial security both at the level of an individual enterprise and at the state level is especially relevant. Considering this, a large number of scientists pay a lot of attention to this issue. In our work, we relied on already existing research in the field of studying the regulatory and legal component of financial security, which is highlighted by Rushchyshyn et al. (2017), who studies it both at the enterprise level and at the level of the entire country.

Special attention should be paid to improving the current legal framework that would protect the interests of the financial system from illegal encroachment and help recover offense damages. All these measures will contribute to raising the level of the state's financial security and will help to integrate the state into the international financial and economic space.

Of course, research is not without limitations and a number of points that should be investigated in further scientific work. So, our research mainly covers the features of financial security in Ukraine. But not all factors and factors that influence financial security were taken into account. In the future, we should expand our research and take into account economic security and make a comparison with other countries with a similar economic environment.

\section{Author contributions}

The authors contributed equally.

\section{Disclosure statement}

The authors do not have any conflict of interest.

\section{References}

Altman, E. (1968). Financial ratios, discriminant analysis and the prediction of corporate bankruptcy. Journal of Finance, 23(4), 589-595.

https://doi.org/10.1111/j.1540-6261.1968.tb00843.x

Arefieva, A., \& Kuzenko, T. (2009). Economic foundations of the formation of financial security. Actual Problems of the Economy, 1(13), 98-103.

Baldacci, E. (2010). Fiscal deficits, public debt and sovereign bond yields. In E. Baldacci \& M. Kumar (Eds.), IMF Working Paper, No. 184. IMF Publications.

Baranovskyi, O. (2004). Financial security in Ukraine (assessment methodology and support mechanisms) [monograph]. Knute.

Bazilyuk, Ya. B. (2020). Organizational mechanisms to reduce threats to economic security in connection with the introduction of measures to prevent the spread of COVID-19 diseases in Ukraine. National Institute for Strategic Research.

https://niss.gov.ua/sites/default/files/2020-04/ekonom-bezpeka-covid.pdf

Beaver, W. (1966). Financial ratios as predictors of failure. Journal of Accounting Research, 4(3), 71-79. https://doi.org/10.2307/2490171

Benigno, G. (2013). Financial crises and macro-prudential. Journal of International Economics, 89(2), 453-470. https://doi.org/10.1016/j.jinteco.2012.06.002

Dmytrenko, Ye. (2011). Place and role of financial and legal responsibility in the mechanism of legal support of Ukraine's financial security. Finance Law, 4(18), 112-119.

Franchuk, V., Omelchuk, O., Melnyk, S., Kelman, M., \& Mykytyuk, O. (2020). Identification the ways of counteraction of the threats to the financial security of high-tech enterprises. Business: Theory and Practice, 21(1), 1-9. https://doi.org/10.3846/btp.2020.11215 
Gebauer, S., Setzer, R., \& Westphal, A. (2017). Corporate debt and investment: a firm level analysis for stressed euro area countries. In ECB Working Paper No. 2101. The European Central Bank (in USA).

https://www.ecb.europa.eu/pub/pdf/scpwps/ecb.wp2101.en.pdf

Greenidge, K. (2012). Threshold effects of sovereign debt: evidence from the Caribbean. In IMF Working Paper No. 12/157. International Monetary Fund. https://doi.org/10.5089/9781475504507.001

Helberg, W. (2011). Normative and instrumental manifestations of the European security strategy in the context of a terrorist threat. Economic Magazine XXI, 11(12), 20-21. http://soskin.info/ea/2011/11-12/201110.html

Hetmanets, O. (2015). Essence and importance of financial and legal responsibility in the budget process. Scientific Bulletin of Uzhhorod National University. Series LAW, 2(30), 101-110.

Kartuzov, E. (2012). Definition of financial security of an enterprise: concept, content, value and functional aspects. Economics and enterprise management. Actual Problems of the Economy, 8(134), 172-181.

Knedlik, T., \& von Schweinitz, G. (2011). Macroeconomic imbalances as indicators for debt crises in Europe. JCMS: Journal of Common Market Studies, 50(5), 726-745. https://doi.org/10.1111/j.1468-5965.2012.02264.x

Krugman, P. (2008). The return of depression economics and the crisis of 2008. W. W. Norton \& Company.

Kulitsky, S. (2020). Problems of development of the Ukrainian economy caused by the COVID-19 coronavirus pandemic in the world, and the search for solutions Ukraine: events, facts, comments. http://nbuviap.gov.ua/index.php?option=com_co ntent \&view $=$ article $\& i d=4890$ : otsinka-perspektiv-rozvit ku-ukrajinskoji-ekonomiki-v-umovakh-pandemiji-covid20\&catid $=64 \&$ Itemid $=376$

Lopez-Claros, A. (2014). Fiscal challenges after the global financial crisis: a survey of key issues. Journal of International Commerce, Economics and Policy, 5(2). https://doi.org/10.1142/S1793993314500045

Lukkezen, J., \& Rojas-Romagosa, H. (2013). Early-warning Indicators for debt sustainability. In CPB Policy Brief, 08. Netherlands Bureau for Economic. https://www.cpb.nl/sites/ default/files/publicaties/download/cpb-presentation-bruegel131008-early-warning-indicators-debt-sustainability.pdf
Malik, A. (2015). The formation of the mechanism of financial security management of the enterprise [Thesis for the degree of $\mathrm{PhD}$ ]. Khmelnitsky.

Marer, P. (2010). The global economic crises: impact on Eastern Europe. Acta Economica, 60(1), 3-33. https://doi.org/10.1556/aoecon.60.2010.1.2

Marsh, P. (2012). The new industrial revolution: consumers, globalization and the end of mass production. Yale University Press.

Radzivil, K. (2011). On the issue of legal support of the state's financial security. Law Bulletin, 4(21), 49-53. https://doi.org/10.18372/2307-9061.21.6595

Reinhart, C. (2011). A decade of debt [Electronic resource]. C. M. Reinhart \& K. S. Rogoff (Eds.). MIT Press. https://doi.org/10.3386/w16827

Roubini, N. (2015). Independence movements: Europe's Paradox. World Economic Forum. https://www.weforum.org/agenda/2015/01/independence-movements-europes-paradox/

Rushchyshyn, N., Nikonenko, U., \& Kostak, Z. (2017). Formation of financial security of the enterprise based on strategic planning. Baltic Journal of Economic Studies, 3(4), 231-237. https://doi.org/10.30525/2256-0742/2017-3-4-231-237

Shevchenko, L. (2009). Economic security of the state: essence and directions of formation [monograph]. Law (in Ukrainian).

Stiglitz, J. (2010). The Stiglitz report: reforming the international monetary and financial systems in the wake of the global crisis. The New Press.

Sylkin, O., Kryshtanovych, M., Zachepa, A., Bilous, S., \& Krasko, A. (2019). Modeling the process of applying anti-crisis management in the system of ensuring financial security of the enterprise. Business: Theory and Practice, 20, 446-455. https://doi.org/10.3846/btp.2019.41

The official website of the Ministry of Finance of Ukraine. (2018). http://www.minfin.gov.ua

Vasyltsiv, T. (2011). Formation of the environment of economic security of the entrepreneurship in Ukraine. Economic Magazine, XXI, 3-4(1), 24-27.

Yermoshenko, M. (2001). Financial security of the state: national interests, real threats, support strategy [Course book]. Knute.

Zaharii, V. (2020). Modeling of monetary parameters of financial security. Effective economics. www.economy.nayka.com.ua 